\title{
INFLUÊNCIA DO MÉTODO DE SÍNTESE NAS CARACTERÍSTICAS ESTRUTURAIS DO TITANATO DE BISMUTO *
}

\author{
Amélia de Santana Cartaxo ${ }^{1}$ \\ Antônio Demouthié de Sales Rolim Esmeraldo² \\ Andrey Gomes Sindeaux ${ }^{3}$ \\ Dário Freire Arrais ${ }^{4}$
}

\begin{abstract}
Resumo
Nos dias atuais, a concorrência comercial estimulada pelas grandes empresas multinacionais tem direcionado 0 rumo das pesquisas cientificas para 0 aperfeiçoamento ou busca de materiais que ofereçam atrativas propriedades físicas ou químicas. O objetivo desse trabalho é analisar a influência dos métodos de síntese nas características estruturais do pó de titanato de bismuto. Foram sintetizadas amostras de titanato de bismuto pelos métodos Pechini e reação de combustão. As amostras sintetizadas foram submetidas as análises de difração de raios $x$, microscopia eletrônica de varredura, área superficial pelo método BET, tamanho de partícula por espalhamento dinâmico de luz, análise térmica pelas técnicas TGA, DTA e DSC. Todos os pós estudados apresentaram morfologia semelhante. As perdas de massas ocorreram em maior escala para as amostras produzidas pelo método de reação de combustão. A fase de titanato de bismuto formou-se em ambos os métodos, entretanto a fase teórica (estequiometria 1:1:3) desejada não foi formada.
\end{abstract}

Palavras-chave: Titanato de bismuto; método Pechini; Reação de combustão.

\section{Abstract}

\section{INFLUENCE OF THE SYNTHESIS METHOD ON STRUCTURAL} CHARACTERISTICS OF BISMUTAN TITANATE

Nowadays, the commercial competition stimulated by the big multinational companies has directed the direction of the scientific researches for the improvement or search of materials that offer attractive physical or chemical properties. The objective of this work is to analyze the influence of the synthesis methods on the structural characteristics of bismuth titanate powder. Samples of bismuth titanate were synthesized by Pechini methods and combustion reaction. The samples were analyzed by $X$ - ray diffraction, scanning electron microscopy, surface area by BET method, particle size by dynamic light scattering, and thermal analysis by TGA, DTA and DSC techniques. All the studied powders presented similar morphology. Mass losses occurred on a larger scale for the samples produced by the combustion reaction method. The bismuth titanate phase was formed in both methods, however the desired theoretical phase (stoichiometry 1: 1:3) was not formed.

Keywords: Bismuth titanate; Pechini method; Combustion reaction.

\footnotetext{
Engenheiro de materiais, bacharelado,,UFC, Juazeiro do Norte, Ceará, Brasil. Engenheiro de materiais, bacharelado,,UFC, Juazeiro do Norte, Ceará, Brasil. Graduando de engenharia metalúrgica, UFC, Fortaleza, Ceará, Brasil.

Graduando de engenharia metalúrgica, UFC, Fortaleza, Ceará, Brasil.
} 


\section{INTRODUÇÃO}

A crescente demanda por novos materiais que apresentem propriedades inovadoras e com potencial para aplicações tecnológicas, principalmente nas áreas de energia e eletrônica, necessitam de desenvolvimento contínuo. Diante disto, os óxidos do tipo Perovskitas têm sido muito estudados, na busca de materiais para aplicações tecnológicas [1, 2].

Diversas técnicas de sínteses, a partir de soluções químicas, podem ser utilizadas para obtenção de pós precursores para formação de Perovskitas com alta pureza, homogeneidade, dimensões nanométricas e estequiometria controlada, com 0 objetivo de obter um melhor controle da microestrutura do material, dentre eles se destacam a síntese Pechini e por reação de combustão[3].

O método Pechini se trata de um processo simples, que se origina de materiais com alta pureza, e leva a obtenção de pós com alta homogeneidade química e alta área de superfície [4]. O método baseia-se na formação de quelatos entre cátions metálicos, em solução aquosa com um ácido hidrocarboxílico. Após a síntese da solução de citrato, é adicionado um poliálcool, para promover a polimerização entre o citrato do íon metálico e o etileno glicol. O aquecimento até temperaturas moderadas (100 ํㅡ), sob atmosfera de ar, causa à reação de esterificação. Durante o aquecimento nessa temperatura ocorre a poliesterificação resultando numa resina polimérica altamente viscosa [5]. O polímero formado apresenta grande homogeneidade na dispersão dos cátions metálicos e um tratamento térmico adequado é realizado para a eliminação da parte orgânica e obtenção da fase desejada [6].

O processo de síntese por reação de combustão tem sido empregado com sucesso para a obtenção de diversos tipos de materiais, visto que possibilitam a obtenção de pós com partículas nanométricas, elevada área superficial e um alto grau de pureza. O método de combustão é autossustentável após o início da reação e atinge altas temperaturas, que garantem a cristalização e formação de pós em curto período de tempo. Sendo assim considerado simples, o método de combustão não envolve muitas etapas e produz pós com elevado grau de pureza, homogeneidade química e quase sempre em escala nanométrica $[7,8]$.

As propriedades físicas das perovskitas são altamente dependentes do método pelo qual foram sintetizadas Em geral, esses materiais são formados em altas temperaturas, entretanto, e os métodos de síntese em baixas temperaturas têm sido utilizados. Baixas temperaturas de síntese resultam em maiores áreas superficiais com menores tamanhos de partícula, tipicamente entre níveis submicro e nano [9].

Com base nesse contexto, este trabalho tem como objetivo desenvolver materiais cerâmicos à base de titanato de bismuto por meio dos métodos de síntese da reação de combustão e Pechini e avaliar a influência destes métodos sobre as características estruturais finais.

\section{MATERIAIS E MÉTODOS}

Para a síntese do titanato de bismuto pelo método Pechini foram utilizados os seguintes reagentes: nitrato de bismuto, citrato de titânio, etilenoglicol e ácido cítrico. Inicialmente, o nitrato de bismuto foi adicionado à água, em seguida o citrato de titânio foi adicionado à mistura. Posteriormente, o ácido cítrico foi adicionado à mistura. Em seguida, foram adicionados ao sistema as quantidades de etilenoglicol e a temperatura foi elevada para $120^{\circ} \mathrm{C}$. Uma vez formada, a resina, foi submetida a 
uma etapa de pirólise, em um forno mufla sob a temperatura de $400^{\circ} \mathrm{C}$ por $8 \mathrm{~h}$. $\mathrm{O}$ produto da etapa da pirólise foi devidamente desagregado em um almofariz, passado em peneira em 325 mesh e submetido à calcinação sob $700^{\circ} \mathrm{C}$ por $3 \mathrm{~h}$.

A síntese pelo método de combustão para preparação do pó de titanato de bismuto envolveu uma mistura contendo íons metálicos, como reagentes oxidantes, e um combustível, como agente redutor, preparada de acordo com a composição estequiométrica estabelecida de acordo com os conceitos da química dos propelentes (jain). Para esta mistura redox foram utilizados nitrato de bismuto, citrato de titânio e ureia. Os reagentes foram misturados em um cadinho de sílica vítrea e submetidos a um pré-aquecimento $120^{\circ} \mathrm{C}$. Durante a combustão a amostra em processo de síntese apresentou o valor máximo de temperatura de $680^{\circ} \mathrm{C}$.

Os pós resultantes de ambos os métodos foram caracterizados por difração de raios $\mathrm{x}$ utilizando um difratômetro de raios $\mathrm{x}$ da marca Rigaku, modelo Miniflex I. Os aspectos morfológicos dos pós, foram analisados por meio de microscopia eletrônica de varredura (MEV), utilizado um microscópio da marca Phillips, modelo XL30 FEG. A análise térmica foi executada através de analizador termogravimétrico da marca TA instruments e modelo SDT 2960, com razão de aquecimento de $20^{\circ} \mathrm{C}$. min $^{-1}$ a um máximo de $1000^{\circ} \mathrm{C}$, sob fluxo de ar sintético com vazão de 110 $\mathrm{mL}$. $\mathrm{min}^{-1}$, utilizando-se um porta-amostra de platina e a massa inicial da amostra foi em torno de $5 \mathrm{mg}$, a distribuição de tamanho de partícula por espalhamento dinâmico de luz (DLS) foi obtida através de um analisador de nanopartículas da marca Shimadzu, modelo IG-1000. E por fim, a área superficial foi calculada pelo método BET, obtida através de um analisador de área superficial por adsorção de nitrogênio da marca Quantachrome Instruments, modelo Quantachrome NovaWin2, version 9.0 NOVA instruments

\section{RESULTADOS E DISCUSSÃO}

\subsection{Difração de raios $x$}

A Figura 1 ilustra os difratogramas de raios $x$ dós pós obtidos por meio do (a) método Pechini, BIP, e por meio da (b) reação de combustão, BIC.

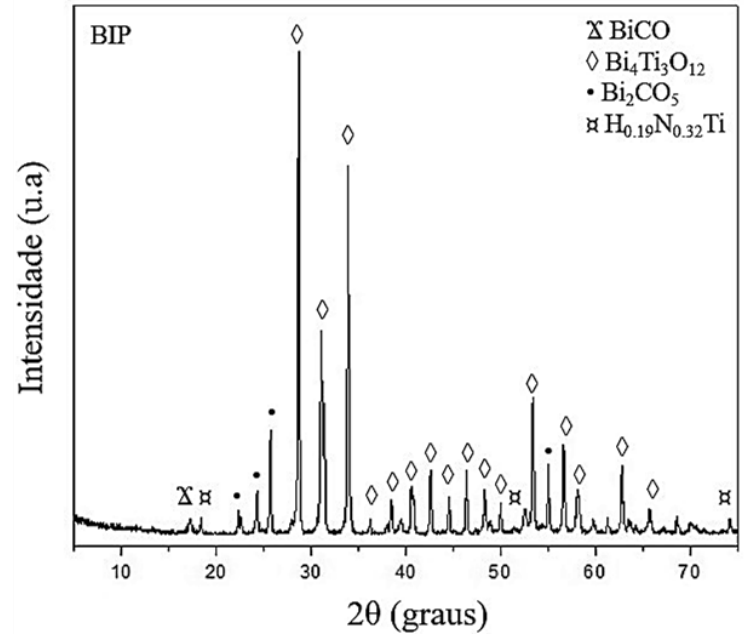

(a)

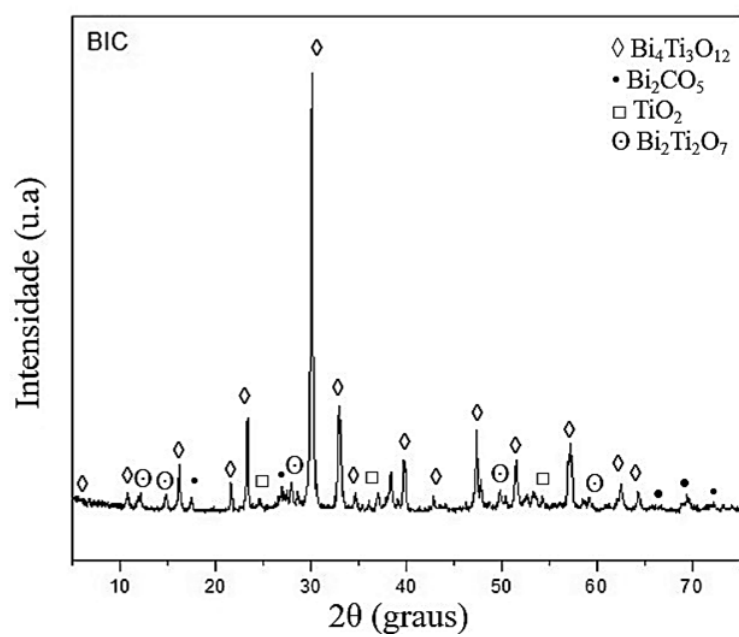

(b)

Figura 1. Difratogramas de raios $x$ dos pós sintetizados pelo método Pechini, (a) BIP, e pelo método de reação de combustão, (b) BIC. 
Para a amostra BIP, Figura 1(a), segundo a natureza dos padrões de difração observados há presença da fase $\mathrm{Bi}_{4} \mathrm{Ti}_{3} \mathrm{O}_{12}$ a qual foi predominante dentre as demais identificadas. Essa fase diferiu da composição teórica, provavelmente pelo excesso de reagentes ou por baixo valor de temperatura de calcinação, entretanto, também representa um titanato de bismuto com célula unitária expandida [10]. A amostra apresentou formação de matéria orgânica residual designada pelas fases: BiCO e $\mathrm{BiCO}_{2}$ e uma fase diferencial chamada de nitreto de titânio hidreto de composição $\mathrm{H}_{0.19} \mathrm{~N}_{0.32} \mathrm{Ti}$.

Para a amostra BIC, Figura 1(b), apresenta-se a formação da fase de titanato de bismuto na composição $\mathrm{Bi}_{4} \mathrm{Ti}_{3} \mathrm{O}_{12}$, o qual teve seu pico $2 \theta$ mais intenso igual a 30.1017 e ocorreu em maior intensidade. Os picos restantes no difratograma foram associados às fases $\mathrm{TiO}_{2}$ (dióxido de titânio), $\mathrm{Bi}_{2} \mathrm{Ti}_{2} \mathrm{O}_{7}$ e $\mathrm{Bi}_{2} \mathrm{CO}_{5}$ (bismutita) as duas primeiras representando fases intermediárias e a segunda impureza na forma de matéria orgânica. Esta fase secundária pode ser atribuída a segregação de uma pequena quantidade de bismuto durante o processo de combustão [11].

Diante disso tanto para as amostras estudadas sintetizadas pelo método Pechini, quanto as sintetizadas pela reação de combustão, foi possível a formação da fase titanato, entretanto, para as últimas houve diminuição da intensidade e da quantidade de picos de difração, evidenciando a importância do tratamento térmico do método Pechini.

\subsection{Análise térmica}

A Figura 2 contêm as curvas de TGA (curva em roxo), DTA (curva em azul) e DSC (curva em vermelho) para as amostras de titanato de bismuto sintetizadas pelos métodos Pechini e pelo método de reação de combustão, respectivamente.

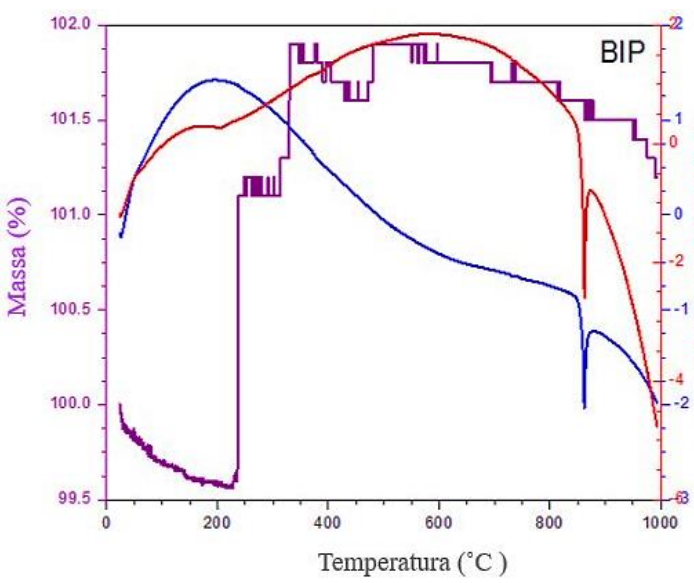

(a)

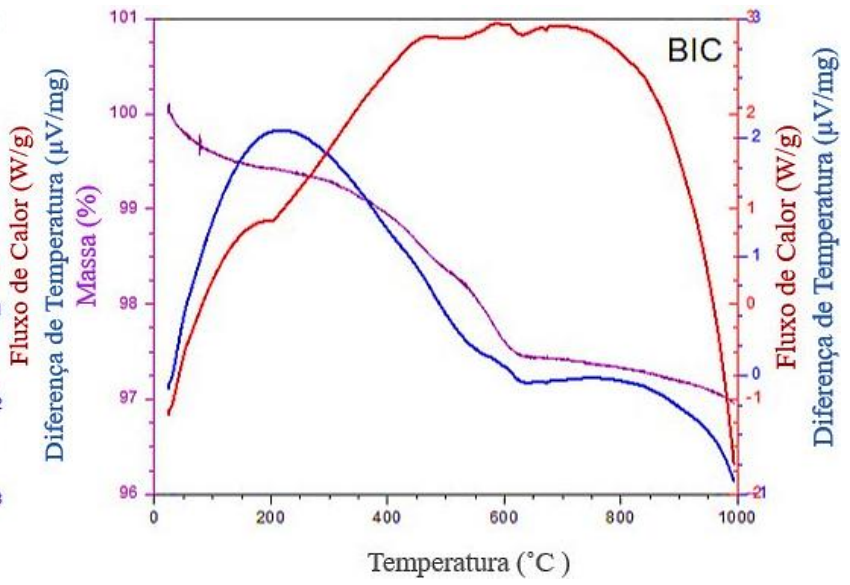

(b)

Figura 2. Curvas de TGA, DTA e DSC para os pós sintetizados pelo método Pechini (a) BIP e pelo método de reação de combustão (b) BIC.

Visualizando as curvas termogravimétricas (TGA) ilustradas na Figura 2(a) e (b) pode-se observar o comportamento térmico dos pós, pela perda de massa em função da temperatura. Nas curvas de análise térmica diferencial (DTA) e de calorimetria diferencial exploratória (DSC) ilustradas na Figura 2(a) e (b) os eventos de liberação de calor (exotérmico) são representados por bandas com a cavidade 
voltada para cima, enquanto os eventos endotérmicos com a cavidade voltada para baixo.

Na Figura 2 (a) pode ser observado que a amostra BIP apresentou uma pequena perda inicial relacionado a água adsorvida, porém houve um provável ganho de massa a partir de $250{ }^{\circ} \mathrm{C}$, acompanhada de um evento exotérmico, comprovado pelas curvas de DTA e DSC (em azul e vermelho, respectivamente). $\mathrm{Em} 850^{\circ} \mathrm{C}$ há um evento endotérmico que pode estar associado com uma mudança de fase.

Na curva de TGA da Figura 2 (b) ocorreram perdas de massa contínuas em toda faixa de temperatura da análise e dessa forma podem ser identificadas três faixas de perda de massa. A primeira perda de massa, que ocorre antes de $300^{\circ} \mathrm{C}$, é atribuída à perda de moléculas voláteis, associadas provavelmente à saída de água residual adsorvida no sistema. O segundo estágio de decomposição, na faixa de $300-650^{\circ} \mathrm{C}$, está associado principalmente ao processo de decomposição do propelente (ureia) devido à combustão incompleta. $E$ finalmente, a terceira perda de massa, acima de aproximadamente $650^{\circ} \mathrm{C}$, refere-se à decomposição de resíduos de carbono e nitratos [12].

Nas curvas de DTA e DSC da Figura 2 (b) pode ser observado eventos que estão em concordância, isto é, foram comprovados por ambas curvas. É possível identificar dois eventos térmicos, o primeiro é mais evidenciado pela da curva de DTA, e ocorreu na faixa de 100 a $500^{\circ} \mathrm{C}$ podendo ser atribuído as primeiras perdas de massa e caracterizado como um processo exotérmico. O segundo ocorre na faixa de $600^{\circ} \mathrm{C}$ a $650^{\circ} \mathrm{C}$ e é classificado como processo endotérmico.

A perda de massa total observada para o pó de titanato de bismuto sintetizado pelo método da combustão apresentou maior valor diante do mesmo produzido através do método Pechini. Evidentemente tal comportamento pode ser explicado pelo fato da exposição do material a um tratamento térmico (etapa de calcinação), no caso do método Pechini, e promover maior eliminação de espécies carbonáceas residuais no produto final.

\subsection{Microscopia eletrônica de varredura}

A morfologia dos das amostras produzidos pelo método Pechini e pelo método de reação de combustão, obtida por microcopia eletrônica de varredura, estão apresentados na Figura 3, com ampliação de 1000x (a,c) e 5000x (b,d).

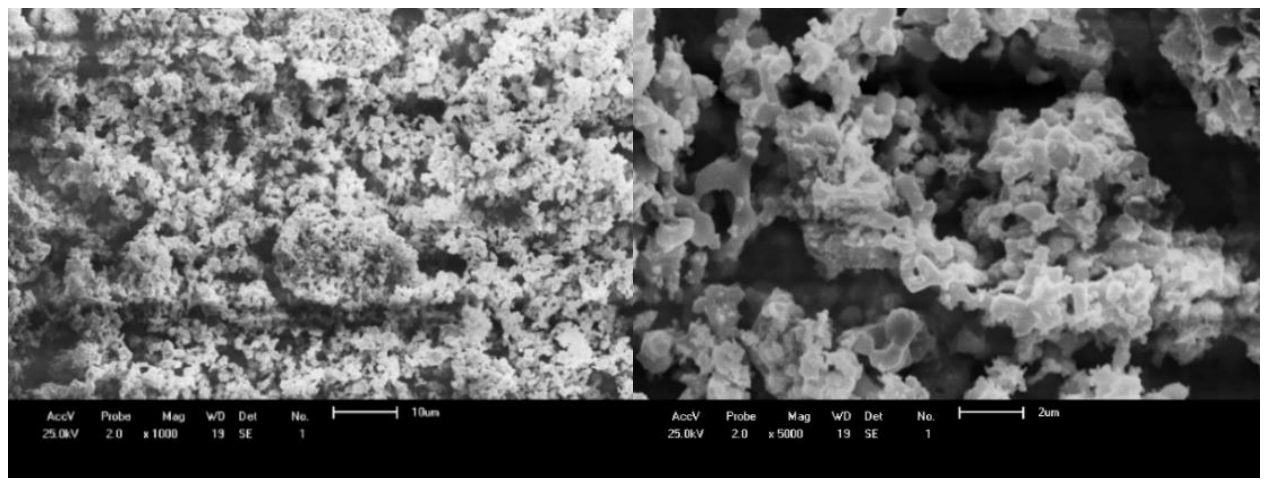

(a)

(b) 

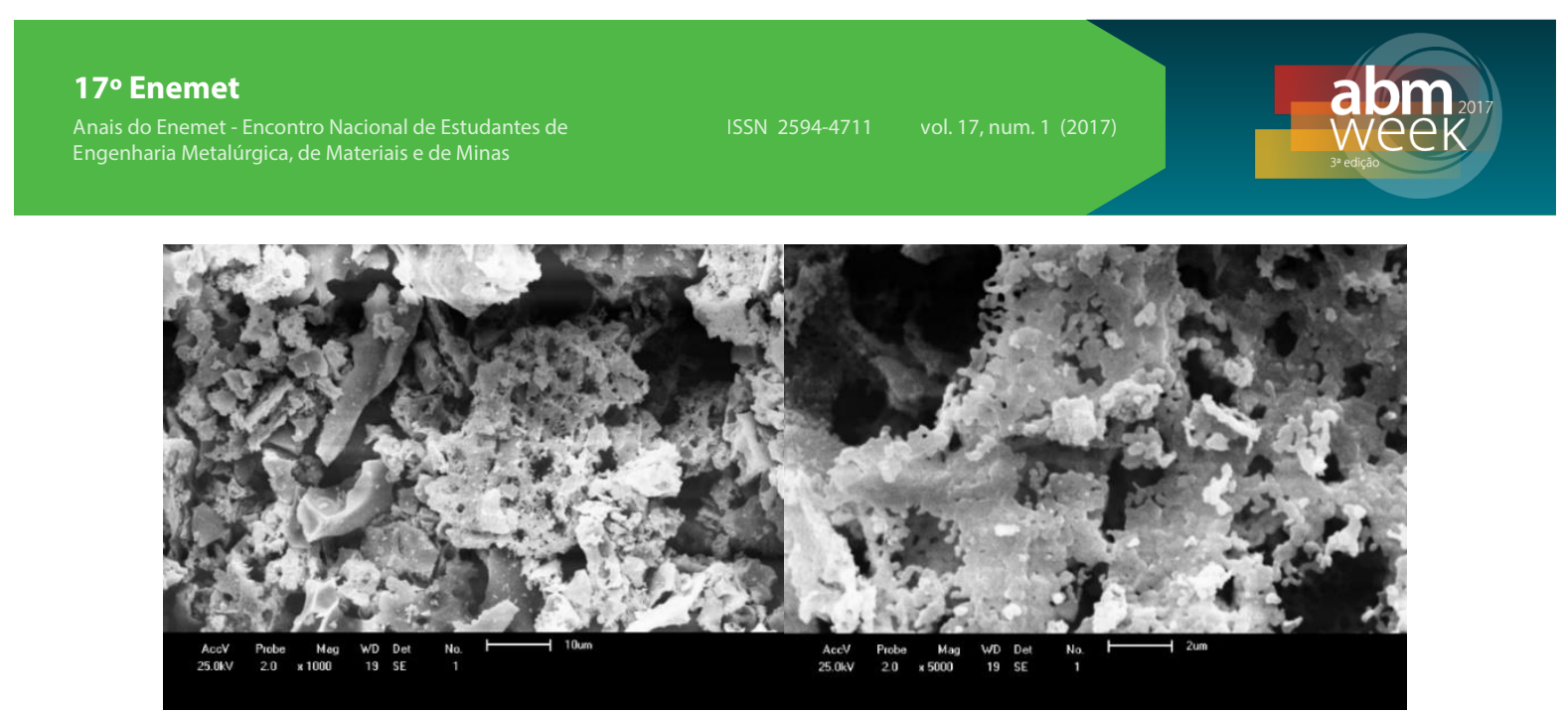

(c)

(d)

Figura 3. Micrografias dos pós sintetizados pelo método Pechini (a): BIP-1000x, (b) BIP-5000x, e pelo método de reação de combustão (c) BIC-1000x, (d) BIC-5000x.

De acordo com as micrografias (Figura 23 (a), (b), (c) e (d)), pode-se observar partículas e aglomerados não uniformes e que o sistema estudado é constituído por aglomerados moles de formato irregular.

A amostra BIP apresentou morfologia na forma de placas ou bastonetes e, adicionalmente, constituída por um grande número de aglomerados [11] esta última característica representada é comum a cerâmicas preparadas por síntese química, como a dos precursores poliméricos (método Pechini).

De acordo com as micrografias, pode-se observar partículas e aglomerados constituído por aglomerados moles, de formato irregular e com uma distribuição heterogênea de tamanhos. Este método possibilita a obtenção de pós que frequentemente aglomeram-se formando partículas secundárias, a fim de minimizar a superfície total ou a energia interfacial do sistema.

\subsection{Espalhamento dinâmico de luz}

Na Figura 4 está ilustrada a distribuição de tamanho de partículas por espalhamento dinâmico de luz referentes aos pós sintetizados pelo método Pechini e pelo método de reação de combustão.

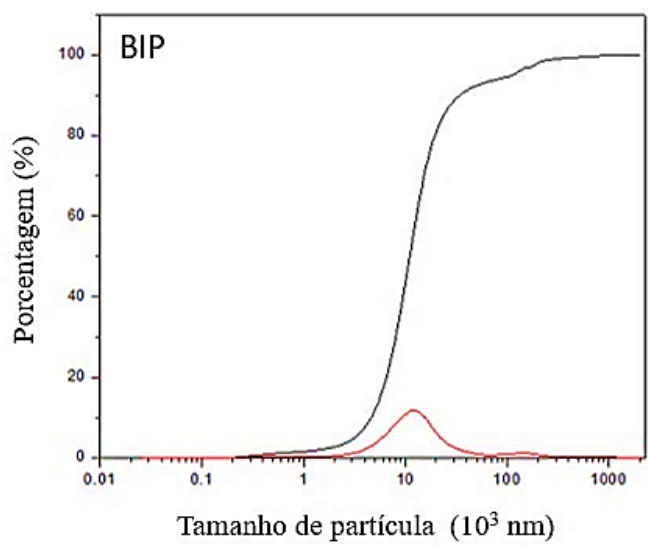

(a)

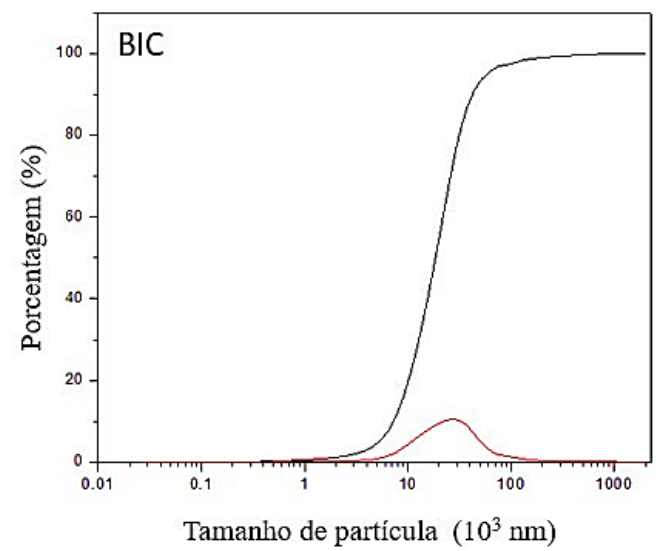

(b)

Figura 4. Tamanho de partículas para os pós sintetizados pelo método Pechini, BIP (a), e pelo método de reação de combustão, BIC (b).

Como pode ser observado na Figura 4 (a) e (b), os pós exibiram distribuição de tamanho de partículas estreita, apresentando uma maior frequência de tamanho 
ocorrendo na faixa entre 10 e $20 \mu \mathrm{m}$ para a amostra BIP, e entre 18 e $23 \mu \mathrm{m}$ para a amostra BIC. Observa-se um diâmetro esférico equivalente médio ( $D_{50}$ ) de $13.14 \mu \mathrm{m}$ para a amostra BIP e $22.06 \mu \mathrm{m}$ para a amostra BIC, respectivamente, os quais encontram-se dentro da faixa de máxima frequência.

\section{5 Área superficial pelo método BET}

Os valores relativos à área superficial dos pós sintetizados, volume e diâmetro dos poros estão apresentados na Tabela 1.

\begin{tabular}{l|c|c|c|c}
\hline AMOSTRA & MÉTODO DE SÍNTESE & $\begin{array}{c}\text { SBET } \\
\left(\mathbf{m}^{2} / \mathbf{g}\right)\end{array}$ & $\mathbf{V p ~}\left(\mathrm{cm}^{3} / \mathbf{g}\right)$ & Dp $(\mathbf{n m})$ \\
\hline BiTiO $_{3}$-BIP & PECHINI & $\mathbf{5 . 3 8 8}$ & $\mathbf{0 . 0 1 1 0 4}$ & $\mathbf{6 . 1 7}$ \\
\hline BiTiO3-BIC $^{\text {COMBUSTÃO }}$ & $\mathbf{1 1 . 0 8}$ & $\mathbf{0 . 0 1 5 8 6}$ & $\mathbf{5 . 1 8 8}$ \\
\hline
\end{tabular}

Tabela 1. Valores relativos a área específica (S $\mathrm{BET})$, volume de poro $(\mathrm{Vp})$, diâmetro médio dos poros (Dp) para as amostras sintetizadas pelo método Pechini e pelo método de reação de combustão.

O valor médio do volume de poros $(\mathrm{Vp})$ e do diâmetro médio de poro $(\mathrm{Dp})$ destas amostras não apresentaram variações significativas em relação aos processos de síntese empregados. Os valores médios dos diâmetros de poro (Dp) das amostras são de dimensões mesoporosas (intervalo entre 2 e 50nm).

Como pode ser observado, o método de síntese influenciou a área superficial obtida, de modo que o método Pechini, para ambos os pós, originou produtos com menores áreas superficial quando comparado com método de reação de combustão. A menor área superficial obtida pelo método Pechini pode ser atribuída à temperatura e ao tempo de calcinação utilizados neste tipo de síntese os quais são maiores quando comparados à reação de combustão, ou seja, no método Pechini o precursor utilizado é submetido a um tratamento térmico mais longo. Adicionalmente, temperaturas elevadas induzem a uma maior energia de ativação favorecendo a formação de partículas maiores e consequentemente redução na área superficial.

\section{CONCLUSÃO}

Foi possível a obtenção dos pós de titanato de bário e de titanato de bismuto, tanto pelo método Pechini quanto pelo método de reação de combustão. Todos os pós estudados apresentaram morfologia semelhante com presença de aglomerados moles e não uniformes e porosos.

De acordo com resultados de difração de raios $\mathrm{x}$ do pó sintetizado pelo método Pechini obteve-se os picos caracterizados por: $\mathrm{Bi}_{4} \mathrm{Ti}_{3} \mathrm{O}_{12}, \mathrm{Bi}_{2} \mathrm{CO}_{5}, \mathrm{BiCO}$ e $\mathrm{H}_{0.19} \mathrm{~N}_{0.32} \mathrm{Ti}$ para a amostra BIP. Paralelamente, o pó sintetizado pelo método de reação de combustão obteve-se os picos caracterizados por: $\mathrm{Bi}_{4} \mathrm{Ti}_{3} \mathrm{O}_{12}, \mathrm{Bi}_{2} \mathrm{CO}_{5}, \mathrm{TiO}_{2}$, $\mathrm{Bi}_{2} \mathrm{Ti}_{2} \mathrm{O}_{7}$ para a amostra BIC.

A análise térmica demostrou as perdas de massa ocorreram em maior escala para as amostras produzidas pelo método de reação de combustão. De acordo com análise de DTA e DSC foi possível detectar eventos térmicos tanto exotérmicos com endotérmicos.

Os resultados obtidos na técnica de DLS permitiram afirmar que as amostras produzidas pelo método Pechini apresentaram uma distribuição de tamanho de 
partículas mais estreita em comparação com as amostras sintetizadas pela reação de combustão.

\section{REFERÊNCIAS}

1 FERNANDES, Felipe de Oliveira. Deposição de filmes de $\mathrm{BiFeO}_{3}$ via spray pirólise e caracterização microestrutural e de propriedades elétricas e ópticas. Tese (doutorado em engenharia) - Escola de engenharia, Universidade Federal do Rio Grande do Sul, Porto Alegre, 2015.

2 STORT, Fernando. Síntese, caracterização estrutural e análise do potencial catalítico do $\mathrm{SrTiO}_{3}$ e das Perovskitas duplas de $\mathrm{Sr}_{1-x} \mathrm{~K}_{x} \mathrm{TiCu}_{\mathrm{x} / 2} \mathrm{O}_{3}$, onde $\mathrm{x}=$ 0,$2 ; 0,3$ e 0,5, na preparação de biodiesel. Dissertação (mestrado em química) - Instituto de Biociências, Letras e Ciências Exatas, Universidade Estadual Paulista, São José do Rio Preto, 2014.

3 SILVEIRA, Valdelice Rodrigues da. Estudo de catalisadores tipo $\mathrm{Ni} / \mathrm{Ce}_{\times} \mathrm{M}_{1-\mathrm{x}} \mathrm{O}_{2}$ ( $\mathrm{M}=\mathrm{Zr}$ ou $\mathrm{Mn}$ ) na reação de oxidação parcial do metano. Tese (doutorado em química) - Universidade Federal do Rio Grande do Norte, Natal, 2010.

4 Lima, U. R.; Bezerra, M. J. O. S.; Pessoa, R. C.; Nasar, M. C.; Nasar, R. S.; Resende, M. C. Síntese da Ferrita de Níquel Zinco pelo método dos Citratos Precursores. $17 \cong$ Congresso Brasileiro de Engenharia e Ciência dos Materiais, 2006, Foz do Iguaçu-PR

5 Lelis, M. F. F. Ferritas dopadas com Níquel ou Cobalto. Síntese, Caracterização e Ação Catalítica na Oxidação do Monóxido de Carbono. Tese - Departamento de Química- Instituto de Ciências Exatas da Universidade Federal de Minas Gerais. 2003.

6 A. N. Simões; D. A. Vieira; M. C. Silva; L. Gama; A. C. F. M. Costa; R. H. G. A. Kiminami. Obtenção de Ferritas Ni-Zn por reação de combustão e método Pechini. Revista Eletrônica de Materiais e Processos, v.4.2 (2009)26-31 ISSN 1809-8797

7 Mimani, T., Alloys J. Comp. 315 (2001) 123-128. 17.

8 Hetting G. F., Worl H., Weiter H. H., Anorg Z., Allg. Chem. 283 (1956) 207.

9 GOODENOUGH, J. B.; ZHOU, J. Varied roles of $\mathrm{Pb}$ in transition-metal $\mathrm{PbMO}_{3}$ perovskites $(\mathrm{M}=\mathrm{Ti}, \mathrm{V}, \mathrm{Cr}, \mathrm{Mn}, \mathrm{Fe}, \mathrm{Ni}, \mathrm{Ru})$. Science and Technology of Advanced Materials, vol. 16, p. 2015

10 AGUIAR, Ederson Carlos de. Desenvolvimento de cerâmicas texturizadas de titanato de bismuto puro e dopado com nióbio. Tese (doutorado em química) Instituto de química, Universidade Estadual Paulista, Araraquara, 2009.

11 MACEDO, Z.S.; FERRARI, C.R.; HERNANDES, A.C. Self-Propagation HighTemperature Synthesis of $\mathrm{Bi}_{4} \mathrm{Ti}_{3} \mathrm{O}_{12}$. Powder Technology, vol. 139(2), p.175179, 2004.

12 SILVA, Cristiane Abrantes da. Técnicas de produção e caracterização de cerâmicas de Zircônia estabilizada com Ítria para aplicação como eletrólito em células a combustível de óxido sólido. Dissertação (mestrado em ciências e tecnologia de processos químicos e bioquímicos. Escola de química Universidade Federal do Rio de Janeiro, Rio de Janeiro, 2008. 